Ciência Florestal, Santa Maria, v. 21, n. 2, p. 193-202, abr.-jun., 2011

ISSN 0103-9954

\title{
PADRÕES PARA GERMINAÇÃO, PUREZA, UMIDADE E PESO DE MIL SEMENTES EM ANÁLISES DE SEMENTES DE ESPÉCIES FLORESTAIS NATIVAS DO RIO GRANDE DO SUL
}

\author{
LIMITS FOR GERMINATION, PURITY, HUMIDITY AND 1000-SEED WEIGHT IN SEED \\ ANALYSIS OF NATIVE TREE SPECIES IN RIO GRANDE DO SUL STATE
}

Fernanda de Oliveira Brüning ${ }^{1}$ Alessandro Dal'Col Lúcioº Marlove Fátima Brião Muniz $^{3}$

\begin{abstract}
RESUMO
O presente trabalho teve como objetivo propor padrões de confiabilidade, em relação à germinação, pureza, peso de mil sementes e grau de umidade das sementes, visando ao controle de qualidade das análises de sementes de espécies florestais. Para tanto, foram utilizados resultados de análises de sementes de espécies florestais em laboratório, a partir do ano de 1997 até 2007, obtidos dos arquivos do Laboratório de Análise de Sementes da Fundação Estadual de Pesquisa Agropecuária do Estado do Rio Grande do Sul. Os padrões de confiabilidade foram construídos com base em uma análise de normalidade dos dados e da utilização do critério do pseudossigma. Os limites se mostraram eficientes no estabelecimento do valor mínimo aceitável para que um lote de sementes seja comercializado, propondo que valores de germinação dentro dos limites de confiabilidade baixos devem ser descartados, pois não apresentam índices germinativos aceitáveis.
\end{abstract}

Palavras-chave: sementes florestais; limites de confiança; critérios de avaliação.

\begin{abstract}
This study had the objective of establishing limits for seed germination, purity, 1000-seed weight and humidity, aiming at the quality control of laboratory seed analysis for forest species. To that end, results of forest species seed analysis from 1997 until 2007 were obtained from the archives of the Laboratory of Seed Analysis of the Agricultural Research Foundation of the State of Rio Grande do Sul. The limits of reliability were constructed from an analysis of data normality and the use of evaluation criteria of Pseudosigma. These limits were effective in the establishment of a minimum acceptable value in order for a plot of seeds to be commercialized, proposing that the germination values inside the low confidence limits must be discarded, because they don't present acceptable germination rates.
\end{abstract}

Keywords: forest seeds; confidence limits; evaluation criteria.

1. Engenheira Florestal, MSc., Técnica da Empresa Brüning Consultoria Agrícola \& Florestal, Bairro Centro, CEP 97400-000, São Pedro do Sul (RS). fernandaoliveira.ef@gmail.com

2. Engenheiro Agrônomo, Dr., Professor do Departamento de Fitotecnia, Centro de Ciências Rurais, Universidade Federal de Santa Maria, Av. Roraima, 1000, CEP 97105-900, Santa Maria (RS). adlucio@smail.ufsm.br

3. Engenheira Agrônoma, Dra ${ }^{\mathrm{a}}$, Professora do Departamento de Defesa Fitossanitária. Campus Universitário, Centro de Ciências Rurais, Universidade Federal de Santa Maria, Av. Roraima, 1000, CEP 97105-900, Santa Maria (RS). marlove@smail.ufsm.br

Recebido para publicação em 20/05/2009 e aceito em 12/08/2010

Ci. Fl., v. 21, n. 2, abr.-jun., 2011 


\section{INTRODUÇÃO}

A qualidade pode ser vista como um padrão de excelência em certos atributos que vão determinar o desempenho da semente. A produção de sementes de alta qualidade engloba vários processos, envolvendo ciência, tecnologia e gestão, o que requer conhecimentos e aptidões específicos (GOULART et al., 2008). Para atender a essas exigências, foram criadas as Regras para Análise de Sementes (RAS). Estas se baseiam naquelas adotadas pela "International Seed Testing Association"- ISTA, em seu "Twentieth International Seed Testing Congres", realizado no Canadá no ano de 1983, e tornadas efetivas em julho de 1985, observando todas as alterações realizadas em tais regras nos congressos subsequentes da ISTA (BRASIL, 1992). A RAS tem por finalidade disponibilizar métodos para análise de sementes, sendo estes obrigatórios nos Laboratórios de Análises de Sementes credenciados no Ministério da Agricultura, Pecuária e Abastecimento (BRASIL, 1992).

A principal finalidade da análise de sementes é determinar a qualidade de um lote e seu consequente valor para a semeadura e armazenamento. A condução de testes de germinação de um grande número de espécies agrícolas cultivadas está prescrita nas Regras para Análise de Sementes (RAS) (BRASIL, 1992). Porém, para as espécies florestais, em especial as nativas, que são comercializadas no Brasil para fins de recomposição florestal, há uma carência de padrões definidos de germinação, pois poucas estão incluídas nas RAS (BRASIL, 1992).

A grande variação biomorfológica dos diferentes tipos de sementes de espécies florestais dificulta o estabelecimento de condições e técnicas adequadas de análise, comprometendo e, muitas vezes, causando insegurança quanto à confiabilidade dos resultados obtidos. Alguns quesitos da qualidade de sementes como porcentagem de pureza, peso de mil sementes e grau de umidade, possuem grande variabilidade nas respostas obtidas quando se averiguam diferentes espécies e, dentro de uma mesma espécie, quanto à procedência das sementes, ao seu lote e ao tempo de armazenamento em câmaras frias (FORTES et al., 2008). Dessa forma, há grande preocupação dos pesquisadores e técnicos em análise de sementes de espécies florestais em realizar estudos que forneçam informações sobre a qualidade destas, a fim de promover padronização, aperfeiçoamento e estabelecimento nos métodos de análise. A falta de padrões estabelecidos para análise de sementes florestais impede que seus resultados sejam utilizados para a fiscalização do comércio e a normatização da produção, bem como para o beneficiamento, armazenamento e distribuição das sementes.

Com a intensificação das fiscalizações dos órgãos ambientais quanto à reposição obrigatória da mata nativa nas propriedades rurais, a produção de sementes de espécies florestais tem aumentado em importância. Dessa forma, o estudo e a padronização dos índices germinativos de espécies nativas possuem grande importância, pois estas são utilizadas sobretudo na Região Sul do Brasil, para fins de recomposição florestal.

Na busca de suprir parte dessas necessidades, foram elaboradas propostas com metodologias para análise de sementes florestais nativas em laboratório (PINÃ-RODRIGUES e VIEIRA, 1988; OLIVEIRA et al. 1989; FIGLIOLIA e PINÃ-RODRIGUES, 1995). A falta de informações sistematizadas sobre os padrões de germinação e outras características fisiológicas das sementes nativas, conforme descrevem Wielewicki et al. (2006), dificulta ainda mais a elaboração de manuais de controle de qualidade dessas sementes. Para o devido estabelecimento desses padrões, faz-se necessário o estudo da distribuição de probabilidade que cada variável está aderindo. Existem diversas funções de distribuição de probabilidade para variáveis aleatórias discretas e contínuas, descritas em Cargnelutti Filho et al. (2004) e Lúcio et al. (2007), cabendo ao pesquisador trabalhar com a hipótese de que a distribuição pode representar adequadamente aquele conjunto de informações (CATALUNHA et al., 2002).

Para verificar a adequação de uma função de distribuição a um conjunto de dados, existem diversos testes não paramétricos, como os de Kolmogorov-Smirnov, Lilliefors (CAMPOS, 1983) e Shapiro-Wilk. Após a identificação da função de distribuição de probabilidade, deve-se determinar os limites na distribuição dos valores observados para as variáveis, adotando um critério, como o de Garcia (1989), o de Lúcio et al. (1999) e o do Pseudossigma (PS) (COSTA et al., 2002). Segundo esses últimos autores, quando há normalidade dos dados, as metodologias de GARCIA (1989) e a do PS são equivalentes.

Nesse sentido, procurando estabelecer valores de germinação para avaliação de lotes de 
sementes, Wielewicki et al. (2006) propuseram padrões mínimos de germinação para espécies florestais nativas do estado do Rio Grande do Sul, utilizando como base o cálculo das médias $(\overline{\mathrm{x}})$ e seus respectivos desvios-padrão (DP). Assim, para Allophylus edulis foi de 79\%; Apuleia leiocarpa, 80\%; Cassia leptophyla, 68\%; Cedrella fissilis, $76 \%$; Enterolobium contortisiliquum, 77\%; Lafoensia pacari, 64\%; Mimosa scabrella, 71\%; Parapiptadenia rigida, 87\%; Peltophorum dubium, 79\%; Jacaranda micrantha, 60\%; Psidium cattleyanum, 74\%; Eugenia involucrata, 51\%; e Eugenia uniflora, $80 \%$. Contudo, esse trabalho não abordou a distribuição de probabilidade desses resultados. Também são citados por Durigan et al. (2002), valores de percentagem de germinação, geralmente superiores a $80 \%$ para Cedrela fissilis Vell.; para Enterolobium contortisiliquum Vell., próximos de 100\%; para Parapiptadenia rigida (Benth.) Brenan, é descrito valor superior a $80 \%$; para Pelthophorum dubium (Spreng.) Taub., valores entre 80 e $90 \%$; e para a espécie Schinus terebinthifolius Raddi, a percentagem de germinação varia em torno de $80 \%$.

Dessa forma, o trabalho teve por objetivo propor limites de classificatórios para germinação de sementes nas análises realizadas em laboratório, com base em uma distribuição de probabilidade, visando à normatização e padronização dos índices germinativos de sementes de espécies florestais.

\section{MATERIAL E MÉTODO}

Foram utilizados os resultados de 2.414 análises de sementes, de 13 espécies florestais nativas, realizadas entre os anos de 1997 e 2007, obtidos dos arquivos do Laboratório de Análise de Sementes da Fundação Estadual de Pesquisa Agropecuária do Estado do Rio Grande do Sul FEPAGRO. As espécies avaliadas foram Allophylus edulis (chal-chal), Apuleia leiocarpa (grápia), Cassia leptophylla (falso-barbatimão), Cassia multijuga (chuva-de-ouro), Cedrela fissilis (cedro), Cupania vernalis (camboatá-vermelho), Enterolobium contortisiliquum (timbaúva), Eugenia involucrata (cerejeira), Jacaranda micrantha (caroba), Lafoensia pacari (dedaleiro), Parapiptadenia rigida (angico-vermelho), Peltophorum dubium (canafístula) e Psidium cattleyanum (araçá).

Em cada análise realizada com essas espécies, foram registradas a análise de pureza
(P\%), o peso de mil sementes (PMS) em gramas, o grau de umidade (U\%) e germinação (G\%), expressa em percentagem de plântulas normais. Partindo da coleta das observações, realizou-se o descarte dos dados daquelas espécies que apresentaram um número de análises igual ou inferior a quatro, por causa da baixa representatividade e confiabilidade dos resultados.

Para todas as variáveis coletadas nas espécies analisadas, foram estimados a Média $(\bar{x})$ e o Desvio-Padrão(DP). Para cada espécie selecionada e cada variável estudada, foram eliminados os valores discrepantes observados em relação à média \pm 2 desvios-padrão. Com os resultados remanescentes foram novamente estimadas a média e o DP, além da Mediana (Md) e do Pseudossigma (Ps), obtido por:

$$
\mathrm{Ps}=\frac{\mathrm{AI}}{1,35}
$$

Em que:

$\mathrm{AI}=$ medida resistente que indica o quanto os dados estão distanciados da mediana;

$1,35=$ valor obtido a partir da distribuição normal, sendo correspondente à distância entre $\mathrm{Q}_{1}$ e $\mathrm{Q}_{3}$, que equivale a $50 \%$ dos dados, deixando $25 \%$ em cada extremidade.

Para verificar se os dados de cada espécie e cada variável seguiram a distribuição normal, foi realizado o teste de Lilliefors, conforme Campos (1983), a 5\% de probabilidade de erro. Os intervalos de classe para cada variável de todas as espécies estudadas foram construídos tomando por base a análise de normalidade dos dados e posterior aplicação do critério do Pseudossigma (COSTA et al., 2002).

\section{RESULTADOS E DISCUSSÃO}

Das espécies analisadas, destaca-se Enterolobium contortisiliquum com a maior percentagem de pureza $(99,78 \%)$ e a menor variabilidade entre as medias de pureza dos lotes, com desvio-padrão (DP) de 0,36. Em contrapartida, as espécies Cedrela fissilis, Lafoensia pacari e Jacaranda micrantha apresentaram as menores purezas, com média de $84,69,89,93$ e $91,28 \%$ respectivamente, e a maior variabilidade entre as médias dos lotes, com os maiores valores dos DP, respectivamente 7,38, 6,09 e 6,65, dentre as espécies analisadas (Tabela 1). A maior média de pureza encontrada para Enterolobium contortisiliquum pode ser explicada pela natureza 
TABELA 1: Média, Desvio-Padrão (DP), Mediana, Pseudossigma (Ps), Número de Análises (N) e Valores Máximo (Máx) e Mínimo (Mín) para a pureza (\%) e o peso de mil sementes (g) de sementes de espécies florestais nativas. Santa Maria, RS, 2009.

TABLE 1: Mean, Standard Deviation (DP), Median, Pseudo-Sigma (Os), number of analysis (N) and maximum (Máx) and minimum (Mín) values for purity (\%) and 1000-seed weight (g) in seeds of native tree species. Santa Maria - RS, 2009.

\begin{tabular}{|c|c|c|c|c|c|c|c|}
\hline \multicolumn{8}{|c|}{ Pureza (\%) } \\
\hline & $\begin{array}{l}\text { Allophylus } \\
\text { edulis }\end{array}$ & $\begin{array}{r}\text { Apuleia } \\
\text { leiocarpa }\end{array}$ & $\begin{array}{c}\text { Cassia } \\
\text { leptophylla }\end{array}$ & $\begin{array}{c}\text { Cassia } \\
\text { multijuga }\end{array}$ & $\begin{array}{l}\text { Cedrela } \\
\text { fissilis }\end{array}$ & $\begin{array}{l}\text { Cupania } \\
\text { vernalis }\end{array}$ & $\begin{array}{c}\text { Enterolobium } \\
\text { contortisiliquum }\end{array}$ \\
\hline Média & 97,99 & 97,97 & 99,34 & 98,57 & 84,69 & 97,74 & 99,78 \\
\hline DP & 1,75 & 1,49 & 0,94 & 1,13 & 7,38 & 0,96 & 0,36 \\
\hline Mediana & 98,96 & 97,94 & 99,67 & 98,83 & 85,98 & 97,61 & 99,92 \\
\hline Ps & 2,00 & 1,91 & 0,31 & 1,69 & 7,47 & 1,01 & 0,22 \\
\hline $\mathrm{N}$ & 42 & 27 & 51 & 32 & 51 & 24 & 59 \\
\hline Máx & 99,64 & 99,81 & 100,00 & 99,96 & 97,69 & 99,31 & 100,00 \\
\hline \multirow[t]{2}{*}{ Mín } & 94,87 & 94,84 & 96,47 & 96,83 & 63,58 & 96,20 & 97,79 \\
\hline & $\begin{array}{c}\text { Eugenia } \\
\text { involucrata }\end{array}$ & $\begin{array}{l}\text { Jacaranda } \\
\text { micrantha }\end{array}$ & $\begin{array}{l}\text { Lafoensia } \\
\text { pacari }\end{array}$ & $\begin{array}{l}\text { Parapiptadenia } \\
\text { rigida }\end{array}$ & $\begin{array}{l}\text { Peltophorum } \\
\text { dubium }\end{array}$ & $\begin{array}{c}\text { Psidium } \\
\text { cattleyanum }\end{array}$ & \\
\hline Média & 98,50 & 91,28 & 89,93 & 97,01 & 98,69 & 97,88 & \\
\hline DP & 1,78 & 6,65 & 6,09 & 2,10 & 1,38 & 1,78 & \\
\hline Mediana & 98,77 & 93,73 & 91,29 & 97,93 & 98,41 & 98,47 & \\
\hline Ps & 1,36 & 4,92 & 6,00 & 1,58 & 1,42 & 1,61 & \\
\hline $\mathrm{N}$ & 21 & 64 & 34 & 54 & 77 & 24 & \\
\hline Máx & 100,00 & 99,90 & 98,76 & 99,71 & 100,0 & 99,85 & \\
\hline Mín & 94,15 & 76,68 & 76,90 & 99,41 & 95,11 & 93,54 & \\
\hline \multicolumn{8}{|c|}{ Peso de mil sementes (g) } \\
\hline & $\begin{array}{l}\text { Allophylus } \\
\text { edulis }\end{array}$ & $\begin{array}{l}\text { Apuleia } \\
\text { leiocarpa }\end{array}$ & $\begin{array}{c}\text { Cassia } \\
\text { leptophylla }\end{array}$ & $\begin{array}{c}\text { Cassia } \\
\text { multijuga }\end{array}$ & $\begin{array}{l}\text { Cedrela } \\
\text { fissilis }\end{array}$ & $\begin{array}{l}\text { Cupania } \\
\text { vernalis }\end{array}$ & $\begin{array}{c}\text { Enterolobium } \\
\text { contortisiliquum }\end{array}$ \\
\hline Média & 41,18 & 92,48 & 143,39 & 10,56 & 28,82 & 330,10 & 234,43 \\
\hline DP & 11,31 & 8,67 & 18,46 & 0,72 & 5,16 & 78,53 & 44,32 \\
\hline Mediana & 41,16 & 88,22 & 139,45 & 10,84 & 29,65 & 307,08 & 235,96 \\
\hline Ps & 7,44 & 11,99 & 19,49 & 1,39 & 6,49 & 84,28 & 47,85 \\
\hline $\mathrm{N}$ & 42 & 27 & 51 & 31 & 51 & 25 & 59 \\
\hline Máx & 65,97 & 106,44 & 178,55 & 11,52 & 39,95 & 460,69 & 346,96 \\
\hline \multirow[t]{2}{*}{ Mín } & 27,47 & 80,17 & 116,12 & 9,56 & 20,03 & 215,91 & 155,40 \\
\hline & $\begin{array}{l}\text { Eugenia } \\
\text { involucrata }\end{array}$ & $\begin{array}{l}\text { Jacaranda } \\
\text { micrantha }\end{array}$ & $\begin{array}{l}\text { Lafoensia } \\
\text { pacari }\end{array}$ & $\begin{array}{l}\text { Parapiptadenia } \\
\text { rigida }\end{array}$ & $\begin{array}{l}\text { Peltophorum } \\
\text { dubium }\end{array}$ & $\begin{array}{c}\text { Psidium } \\
\text { cattleyanum }\end{array}$ & \\
\hline Média & 352,36 & 6,71 & 18,05 & 25,37 & 49,74 & 16,15 & \\
\hline DP & 72,50 & 0,95 & 0,94 & 3,41 & 4,10 & 5,43 & \\
\hline Mediana & 377,45 & 6,92 & 17,81 & 24,57 & 49,71 & 15,77 & \\
\hline Ps & 109,73 & 0,73 & 0,93 & 3,10 & 3,29 & 6,93 & \\
\hline $\mathrm{N}$ & 20 & 64 & 34 & 53 & 75 & 24 & \\
\hline Máx & 434,16 & 8,06 & 21,24 & 32,65 & 61,75 & 24,93 & \\
\hline Mín & 217,94 & 0,66 & 16,28 & 18,86 & 39,81 & 7,61 & \\
\hline
\end{tabular}

Ci. Fl., v. 21, n. 2, abr.-jun., 2011 
da cobertura das sementes dessa espécie, com tegumento mais duro, que diminui a ocorrência de quebra, reduzindo a presença de material inerte na amostra. Já a menor média de pureza encontrada para Cedrela fissilis e Jacaranda micrantha, devese ao fato dessas espécies possuírem sementes com estruturas em forma de alas, que facilitam a dispersão anemocórica e quebram-se facilmente, sendo classificadas como impurezas dentro da amostra.

$\mathrm{Na}$ variável peso de mil sementes, Eugenia involucrata apresentou maior média, com 352,36 g. As sementes dessa espécie apresentam elevada quantidade de água no seu interior, o que as torna mais densas e pesadas podendo explicar o maior peso de mil sementes apresentado e segundo Barbedo et al. (1998), várias espécies brasileiras de Eugenia apresentam elevado teor de água (entre 40 e $70 \%$ ), o que influencia nas avaliações de peso das sementes. Em segundo lugar, destacou-se a espécie Cupania vernalis com 330,10g em mil sementes, seguida pelo Enterolobium contortisiliquum (Tabela 1). Percebe-se também que as espécies com maiores médias de peso de mil sementes foram as que apresentaram também os maiores valores do DP, confirmando que a diferença entre lotes, nesse aspecto, é por causa da produção, vigor da plantamãe e característica das matrizes.

Normalmente, as sementes de espécies nativas apresentam maior variabilidade de características em relação às espécies exóticas. Isso pode ocorrer em razão da grande diversidade genética existente entre essas espécies, sobre as quais, diferentemente das exóticas, não foram realizados estudos de melhoramento genético e das características físicas e morfológicas.

Das espécies analisadas, a maioria possui sementes tolerantes à dessecação, as quais, de acordo com Vieira et al. (2001), podem ser armazenadas com um baixo teor de umidade e temperatura, mantendo sua viabilidade por um maior período de tempo. Apenas Eugenia involucrata e Cupania vernalis possuem sementes sensíveis à dessecação e estas apresentaram elevados teores de umidade, com 47,26 e 32, 69\%, respectivamente, (Tabela 2), diferenciando-se das demais espécies estudadas, pois, em função de sua característica, não sofrem secagem natural na planta-mãe e são liberadas ou coletadas com elevado teor de umidade (VIEIRA et al., 2001).

Algumas espécies apresentaram elevado percentual de germinação, entre as quais se destacou
Parapiptadenia rigida, com média de 94,14\% e reduzido DP (Tabela 2). A germinação de lotes armazenados está muito relacionada à capacidade da semente em suportar a dessecação e baixas temperaturas no armazenamento ou ser intolerante a essas situações. Segundo descrevem Wielewicki et al. (2006), a espécie Parapiptadenia rigida possui sementes com características tolerantes à dessecação, e este pode ser o fator determinante para as altas taxas germinativas apresentadas. Já a menor média de germinação foi apresentada por Cupania vernalis, com 39,50\% e o maior valor do DP (Tabela 2). Isso pode relacionar-se ao fato de essa espécie ser sensível à dessecação, necessitando de valores elevados de umidade para manutenção de sua viabilidade e sobrevivência. Também, as sementes com alto teor de umidade deterioramse mais rápido em função da alta incidência de fungos ao longo do tempo de armazenamento, além da diversidade genética existente entre os lotes de sementes de espécies nativas, o que gera a grande variabilidade entre lotes dessa espécie.

Pelo teste de Lilliefors, verificou-se que das 13 espécies analisadas, 46,14\% apresentaram normalidade dos dados para as variáveis pureza, umidade e germinação e $38,46 \%$, para a variável peso de mil sementes (Tabela 3). Resultados que podem estar relacionados à grande variabilidade genética das espécies florestais nativas, as quais, em maioria, ainda não foram melhoradas geneticamente. Dentre as espécies, a única que apresentou aderência dos dados à distribuição normal para as quatro variáveis analisadas foi Eugenia involucrata, seguida por Cedrela fissilis e Lafoensia pacari com três aderências em quatro das variáveis avaliadas.

No momento de interpretação dos padrões propostos, é importante ressaltar que lotes de sementes com valores de germinação (\%) dentro dos limites baixos (B) (Tabelas 4 a 7), devem ser vistos com ressalvas no momento da comercialização pois, de acordo com Wielewicki et al. (2006), valores inferiores ao proposto poderiam expressar problemas de qualidade do lote, levando-se em consideração o potencial germinativo da espécie. O valor mínimo aceitável para que um lote de sementes seja comercializado deve estar dentro do limite de confiabilidade médio (M). Já os valores desejáveis para certificação e comercialização de lotes de sementes devem estar dentro do limite alto (A). 
TABELA 2: Média, Desvio Padrão (DP), Mediana, Pseudo-sigma (Ps), Número de Análises (N) e Valores Máximo (Máx) e Mínimo (Mín) para a umidade (\%) e a germinação (\%) de sementes de espécies florestais nativas. Santa Maria, RS, 2009.

TABLE 2: Mean, Standard Deviation (DP), Median, Pseudo-Sigma (Os), number of analysis (N) and maximum (Máx) and minimum (Mín) values for humidity (\%) and germination (\%) in seeds of native tree species. Santa Maria - RS, 2009.

\begin{tabular}{|c|c|c|c|c|c|c|c|}
\hline \multicolumn{8}{|c|}{ Umidade (\%) } \\
\hline & $\begin{array}{l}\text { Allophylus } \\
\text { edulis }\end{array}$ & $\begin{array}{l}\text { Apuleia } \\
\text { leiocarpa }\end{array}$ & $\begin{array}{c}\text { Cassia } \\
\text { leptophylla }\end{array}$ & $\begin{array}{c}\text { Cassia } \\
\text { multijuga }\end{array}$ & $\begin{array}{l}\text { Cedrela } \\
\text { fissilis }\end{array}$ & $\begin{array}{l}\text { Cupania } \\
\text { vernalis }\end{array}$ & $\begin{array}{c}\text { Enterolobium } \\
\text { contortisiliquum }\end{array}$ \\
\hline Média & 20,67 & 11,28 & 10,73 & 10,62 & 9,59 & 32,69 & 8,91 \\
\hline DP & 9,49 & 1,05 & 1,66 & 1,34 & 1,53 & 4,99 & 2,66 \\
\hline Mediana & 18,99 & 11,47 & 10,64 & 10,19 & 9,36 & 32,35 & 8,21 \\
\hline Ps & 14,39 & 1,13 & 1,90 & 0,67 & 1,60 & 7,29 & 2,17 \\
\hline $\mathrm{N}$ & 32 & 17 & 43 & 24 & 43 & 19 & 48 \\
\hline Máx & 34,84 & 12,76 & 14,86 & 14,07 & 13,57 & 39,57 & 19,73 \\
\hline \multirow[t]{2}{*}{ Mín } & 7,24 & 8,89 & 8,29 & 8,76 & 6,71 & 24,59 & 5,72 \\
\hline & $\begin{array}{c}\text { Eugenia } \\
\text { involucrata }\end{array}$ & $\begin{array}{l}\text { Jacaranda } \\
\text { micrantha }\end{array}$ & $\begin{array}{l}\text { Lafoensia } \\
\text { pacari }\end{array}$ & $\begin{array}{l}\text { Parapiptadenia } \\
\text { rigida }\end{array}$ & $\begin{array}{l}\text { Peltophorum } \\
\text { dubium }\end{array}$ & $\begin{array}{c}\text { Psidium } \\
\text { cattleyanum }\end{array}$ & \\
\hline Média & 47,26 & 7,80 & 12,11 & 13,33 & 9,29 & 14,24 & \\
\hline DP & 4,73 & 1,52 & 2,45 & 3,13 & 1,04 & 10,00 & \\
\hline Mediana & 47,72 & 7,63 & 11,53 & 13,16 & 9,09 & 11,84 & \\
\hline Ps & 3,89 & 1,35 & 1,72 & 2,77 & 1,21 & 1,20 & \\
\hline $\mathrm{N}$ & 18 & 49 & 21 & 42 & 73 & 15 & \\
\hline Máx & 54,77 & 11,85 & 19,24 & 23,46 & 13,45 & 55,32 & \\
\hline Mín & 35,41 & 4,80 & 9,50 & 8,94 & 7,42 & 10,41 & \\
\hline \multicolumn{8}{|c|}{ Germinação (\%) } \\
\hline & $\begin{array}{l}\text { Allophylus } \\
\text { edulis }\end{array}$ & $\begin{array}{l}\text { Apuleia } \\
\text { leiocarpa }\end{array}$ & $\begin{array}{c}\text { Cassia } \\
\text { leptophylla }\end{array}$ & $\begin{array}{c}\text { Cassia } \\
\text { multijuga }\end{array}$ & $\begin{array}{l}\text { Cedrela } \\
\text { fissilis }\end{array}$ & $\begin{array}{l}\text { Cupania } \\
\text { vernalis }\end{array}$ & $\begin{array}{c}\text { Enterolobium } \\
\text { contortisiliquum }\end{array}$ \\
\hline Média & 67,31 & 70,30 & 57,38 & 66,23 & 84,07 & 39,50 & 89,05 \\
\hline DP & 19,02 & 22,15 & 20,74 & 23,68 & 11,22 & 24,26 & 10,00 \\
\hline Mediana & 72,00 & 81,00 & 60,75 & 73,00 & 88,00 & 33,00 & 93,00 \\
\hline Ps & 24,44 & 21,30 & 18,00 & 27,41 & 12,59 & 25,00 & 9,63 \\
\hline $\mathrm{N}$ & 42 & 27 & 55 & 32 & 55 & 25 & 58 \\
\hline Máx & 94,00 & 96,50 & 95,00 & 94,00 & 97,00 & 89,50 & 99,00 \\
\hline \multirow[t]{2}{*}{ Mín } & 36,00 & 23,00 & 11,00 & 10,75 & 58,00 & 9,50 & 61,00 \\
\hline & $\begin{array}{c}\text { Eugenia } \\
\text { involucrata }\end{array}$ & $\begin{array}{l}\text { Jacaranda } \\
\text { micrantha }\end{array}$ & $\begin{array}{l}\text { Lafoensia } \\
\quad \text { pacari }\end{array}$ & $\begin{array}{l}\text { Parapiptadenia } \\
\text { rigida }\end{array}$ & $\begin{array}{l}\text { Peltophorum } \\
\text { dubium }\end{array}$ & $\begin{array}{c}\text { Psidium } \\
\text { cattleyanum }\end{array}$ & \\
\hline Média & 81,76 & 85,28 & 76,44 & 94,14 & 82,81 & 76,00 & \\
\hline DP & 9,59 & 9,12 & 12,97 & 5,31 & 10,72 & 9,06 & \\
\hline Mediana & 81,00 & 88,00 & 80,00 & 96,00 & 87 & 76,50 & \\
\hline Ps & 12,22 & 6,85 & 13,33 & 6,02 & 12,59 & 9,21 & \\
\hline $\mathrm{N}$ & 23 & 63 & 34 & 54 & 77 & 23 & \\
\hline Max. & 97,00 & 98,25 & 95,00 & 100,00 & 98,00 & 91,00 & \\
\hline Mín. & 66,50 & 58,25 & 38,00 & 80,00 & 59,00 & 50,25 & \\
\hline
\end{tabular}

Ci. Fl., v. 21, n. 2, abr.-jun., 2011 
Padrões para germinação, pureza, umidade e peso de mil sementes em análises de sementes ...

TABELA 3: Aderência à distribuição normal dos resultados nas análises de sementes de espécies nativas para pureza (\%), peso de mil sementes (PMS) $(\mathrm{g})$, umidade (\%) e germinação $(\mathrm{G} \%)$. Santa Maria, RS, 2009.

TABLE 3: Adherence to the normal distribution of the results in analyses of seeds of native tree species for purity (\%), 1000-seed weight (PMS) (g), humidity (\%) and germination . Santa Maria RS, 2009.

\begin{tabular}{c|c|c|c|c|c|c|c}
\hline Variáveis & $\begin{array}{c}\text { Allophylus } \\
\text { edulis }\end{array}$ & $\begin{array}{c}\text { Apuleia } \\
\text { leiocarpa }\end{array}$ & $\begin{array}{c}\text { Cassia } \\
\text { leptophylla }\end{array}$ & $\begin{array}{c}\text { Cassia } \\
\text { multijuga }\end{array}$ & $\begin{array}{c}\text { Cedrela } \\
\text { fissilis }\end{array}$ & $\begin{array}{c}\text { Cupania } \\
\text { vernalis }\end{array}$ & $\begin{array}{c}\text { Enterolobium } \\
\text { contortisiliquum }\end{array}$ \\
\hline Pureza & $\mathrm{NN}$ & $\mathrm{N}$ & $\mathrm{NN}$ & $\mathrm{N}$ & $\mathrm{N}$ & $\mathrm{N}$ & $\mathrm{NN}$ \\
PMS & $\mathrm{NN}$ & $\mathrm{NN}$ & $\mathrm{NN}$ & $\mathrm{NN}$ & $\mathrm{N}$ & $\mathrm{NN}$ & $\mathrm{N}$ \\
Umidade & $\mathrm{NN}$ & $\mathrm{N}$ & $\mathrm{N}$ & $\mathrm{NN}$ & $\mathrm{N}$ & $\mathrm{NN}$ & $\mathrm{NN}$ \\
$\mathrm{G}$ & $\mathrm{N}$ & $\mathrm{NN}$ & $\mathrm{N}$ & $\mathrm{N}$ & $\mathrm{NN}$ & $\mathrm{NN}$ & $\mathrm{NN}$ \\
\hline \multirow{2}{*}{ Variáveis } & Eugenia & Jacaranda & Lafoensia & Parapiptadenia & Peltophorum & Psidium & \\
\hline Pureza & $\mathrm{N}$ & $\mathrm{NN}$ & $\mathrm{N}$ & $\mathrm{NN}$ & $\mathrm{NN}$ & $\mathrm{NN}$ & \\
PMS & $\mathrm{N}$ & $\mathrm{NN}$ & $\mathrm{N}$ & $\mathrm{NN}$ & $\mathrm{N}$ & $\mathrm{NN}$ & \\
Umidade & $\mathrm{N}$ & $\mathrm{N}$ & $\mathrm{NN}$ & $\mathrm{N}$ & $\mathrm{NN}$ & $\mathrm{NN}$ & \\
$\mathrm{G}$ & $\mathrm{N}$ & $\mathrm{NN}$ & $\mathrm{N}$ & $\mathrm{NN}$ & $\mathrm{NN}$ & $\mathrm{N}$ & \\
\hline
\end{tabular}

Em que: $\mathrm{N}=$ resultados da variável se aderem à distribuição normal pelo teste de Lilliefors a $5 \%$ de probabilidade de erro; $\mathrm{NN}$ = resultados da variável não se aderem à distribuição normal pelo teste de Lilliefors a $5 \%$ de probabilidade de erro.

TABELA 4: Limites Baixo (B), Médio (M), Alto (A) e Muito Alto (MA) para a pureza (\%) de sementes de espécies florestais nativas. Santa Maria, RS. 2009.

TABLE 4: Low (B), Medium (M), High (A) and Very HIGH (MA) Limits for purity (\%) in seeds of native tree species. Santa Maria - RS, 2009.

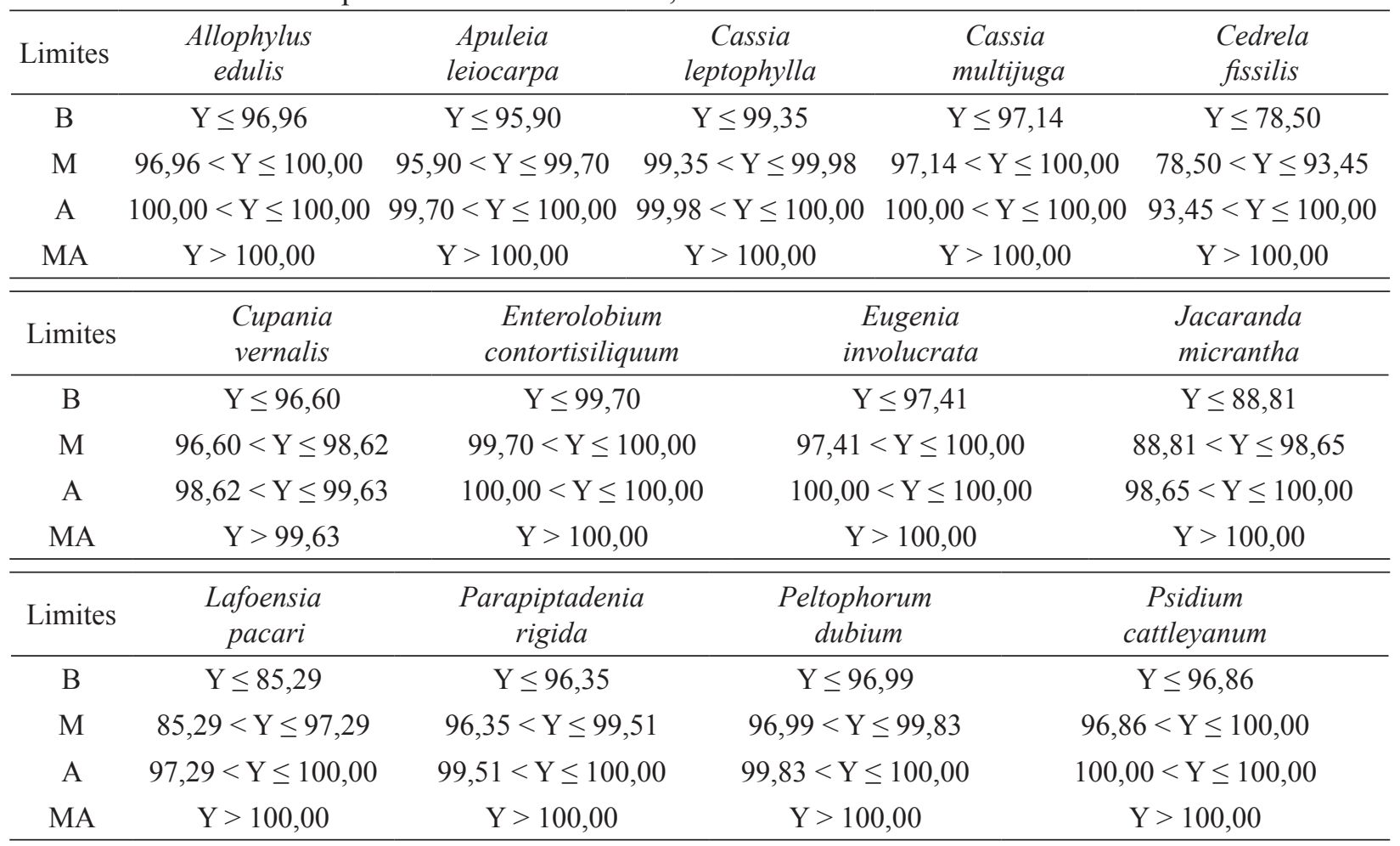


TABELA 5: Limites Baixo (B), Médio (M), Alto (A) e Muito Alto (MA) para o peso de mil sementes (g) de sementes de espécies florestais nativas. Santa Maria, RS. 2009.

TABLE 5: Low (B), Medium (M), High (A) and Very High (MA) Limits for 1000-seed weight (g) in seeds of native tree species. Santa Maria - RS, 2009.

\begin{tabular}{cccccc}
\hline Limites & $\begin{array}{c}\text { Allophylus } \\
\text { edulis }\end{array}$ & $\begin{array}{c}\text { Apuleia } \\
\text { leiocarpa }\end{array}$ & $\begin{array}{c}\text { Cassia } \\
\text { leptophylla }\end{array}$ & $\begin{array}{c}\text { Cassia } \\
\text { multijuga }\end{array}$ & $\begin{array}{c}\text { Cedrela } \\
\text { fissilis }\end{array}$ \\
\hline $\mathrm{B}$ & $\mathrm{Y} \leq 31,71$ & $\mathrm{Y} \leq 76,23$ & $\mathrm{Y} \leq 119,96$ & $\mathrm{Y} \leq 9,45$ & $\mathrm{Y} \leq 23,16$ \\
$\mathrm{M}$ & $31,71<\mathrm{Y} \leq 48,60$ & $76,23<\mathrm{Y} \leq 100,21$ & $119,96<\mathrm{Y} \leq 158,93$ & $9,45<\mathrm{Y} \leq 12,23$ & $23,16<\mathrm{Y} \leq 36,14$ \\
$\mathrm{~A}$ & $48,60<\mathrm{Y} \leq 56,04$ & $100,21<\mathrm{Y} \leq 112,19$ & $158,93<\mathrm{Y} \leq 178,42$ & $12,23<\mathrm{Y} \leq 13,62$ & $36,14<\mathrm{Y} \leq 42,63$ \\
$\mathrm{MA}$ & $\mathrm{Y}>56,04$ & $\mathrm{Y}>112,19$ & $\mathrm{Y}>178,42$ & $\mathrm{Y}>13,62$ & $\mathrm{Y}>42,63$ \\
\hline \hline \multirow{2}{*}{ Limites } & Cupania & Enterolobium & Eugenia & Jacaranda \\
& vernalis & contortisiliquum & involucrata & micrantha \\
\hline $\mathrm{B}$ & $\mathrm{Y} \leq 222,81$ & $\mathrm{Y} \leq 188,10$ & $\mathrm{Y} \leq 267,72$ & $\mathrm{Y} \leq 6,20$ \\
$\mathrm{M}$ & $222,81<\mathrm{Y} \leq 301,36$ & $188,10<\mathrm{Y} \leq 283,81$ & $267,72<\mathrm{Y} \leq 487,18$ & $6,20<\mathrm{Y} \leq 7,65$ \\
$\mathrm{~A}$ & $391,36<\mathrm{Y} \leq 475,64$ & $283,81<\mathrm{Y} \leq 331,67$ & $487,18<\mathrm{Y} \leq 596,91$ & $7,65<\mathrm{Y} \leq 8,38$ \\
$\mathrm{MA}$ & $\mathrm{Y}>475,64$ & $\mathrm{Y}>311,67$ & $\mathrm{Y}>596,91$ & $\mathrm{Y}>8,38$ \\
\hline \hline \multirow{2}{*}{ Limites } & Lafoensia & Parapiptadenia & Peltophorum & Psidium \\
& pacari & rigida & dubium & cattleyanum \\
\hline $\mathrm{B}$ & $\mathrm{Y} \leq 16,88$ & $\mathrm{Y} \leq 21,46$ & $\mathrm{Y} \leq 46,42$ & $\mathrm{Y} \leq 8,84$ \\
$\mathrm{M}$ & $16,88<\mathrm{Y} \leq 18,74$ & $21,46<\mathrm{Y} \leq 27,67$ & $46,42<\mathrm{Y} \leq 53,00$ & $8,84<\mathrm{Y} \leq 22,70$ \\
$\mathrm{~A}$ & $18,74<\mathrm{Y} \leq 19,67$ & $27,67<\mathrm{Y} \leq 30,78$ & $53,00<\mathrm{Y} \leq 56,29$ & $22,70<\mathrm{Y} \leq 29,63$ \\
$\mathrm{MA}$ & $\mathrm{Y}>19,67$ & $\mathrm{Y}>30,78$ & $\mathrm{Y}>56,29$ & $\mathrm{Y}>29,63$ \\
\hline
\end{tabular}

TABELA 6: Limites Baixo (B), Médio (M), Alto (A) e Muito Alto (MA) para a umidade (\%) de sementes de espécies florestais nativas. Santa Maria, RS. 2009.

TABLE 6: Low (B), Medium (M), High (A) and Very High (MA) Limits for humidity (\%) in seeds of native tree species. Santa Maria - RS, 2009.

\begin{tabular}{cccccc}
\hline Limites & $\begin{array}{c}\text { Allophylus } \\
\text { edulis }\end{array}$ & $\begin{array}{c}\text { Apuleia } \\
\text { leiocarpa }\end{array}$ & $\begin{array}{c}\text { Cassia } \\
\text { leptophylla }\end{array}$ & $\begin{array}{c}\text { Cassia } \\
\text { multijuga }\end{array}$ & $\begin{array}{c}\text { Cedrela } \\
\text { fissilis }\end{array}$ \\
\hline $\mathrm{B}$ & $\mathrm{Y} \leq 4,60$ & $\mathrm{Y} \leq 10,34$ & $\mathrm{Y} \leq 8,74$ & $\mathrm{Y} \leq 9,52$ & $\mathrm{Y} \leq 7,76$ \\
$\mathrm{M}$ & $4,60<\mathrm{Y} \leq 33,38$ & $10,34<\mathrm{Y} \leq 12,60$ & $8,74<\mathrm{Y} \leq 12,54$ & $9,52<\mathrm{Y} \leq 10,86$ & $7,76<\mathrm{Y} \leq 10,96$ \\
$\mathrm{~A}$ & $33,38<\mathrm{Y} \leq 47,78$ & $12,60<\mathrm{Y} \leq 13,72$ & $12,54<\mathrm{Y} \leq 14,44$ & $10,86<\mathrm{Y} \leq 11,52$ & $10,96<\mathrm{Y} \leq 12,56$ \\
$\mathrm{MA}$ & $\mathrm{Y}>47,78$ & $\mathrm{Y}>13,72$ & $\mathrm{Y}>14,44$ & $\mathrm{Y}>11,52$ & $\mathrm{Y}>12,56$ \\
\hline \hline \multirow{2}{*}{ Limites } & Cupania & Enterolobium & Eugenia & Jacaranda \\
& vernalis & contortisiliquum & involucrata & micrantha \\
\hline $\mathrm{B}$ & $\mathrm{Y} \leq 25,06$ & $\mathrm{Y} \leq 6,05$ & $\mathrm{Y} \leq 43,83$ & $\mathrm{Y} \leq 6,28$ \\
$\mathrm{M}$ & $25,06<\mathrm{Y} \leq 39,64$ & $6,05<\mathrm{Y} \leq 10,38$ & $43,83<\mathrm{Y} \leq 51,61$ & $6,28<\mathrm{Y} \leq 8,98$ \\
$\mathrm{~A}$ & $39,64<\mathrm{Y} \leq 46,93$ & $10,38<\mathrm{Y} \leq 12,55$ & $51,61<\mathrm{Y} \leq 55,50$ & $8,98<\mathrm{Y} \leq 10,33$ \\
$\mathrm{MA}$ & $\mathrm{Y}>46,93$ & $\mathrm{Y}>12,55$ & $\mathrm{Y}>50,03$ & $\mathrm{Y}>10,33$ \\
\hline \hline \multirow{2}{*}{ Limites } & Lafoensia & Parapiptadenia & Peltophorum & Psidium \\
& pacari & rigida & dubium & cattleyanum \\
\hline $\mathrm{B}$ & $\mathrm{Y} \leq 9,81$ & $\mathrm{Y} \leq 10,39$ & $\mathrm{Y} \leq 7,88$ & $\mathrm{Y} \leq 10,64$ \\
$\mathrm{M}$ & $9,81<\mathrm{Y} \leq 13,25$ & $10,39<\mathrm{Y} \leq 15,93$ & $7,88<\mathrm{Y} \leq 10,30$ & $10,64<\mathrm{Y} \leq 13,04$ \\
$\mathrm{~A}$ & $13,25<\mathrm{Y} \leq 14,96$ & $15,93<\mathrm{Y} \leq 18,70$ & $10,30<\mathrm{Y} \leq 11,51$ & $13,04<\mathrm{Y} \leq 14,25$ \\
$\mathrm{MA}$ & $\mathrm{Y}>14,96$ & $\mathrm{Y}>18,70$ & $\mathrm{Y}>11,51$ & $\mathrm{Y}>14,25$ \\
\hline
\end{tabular}

Ci. Fl., v. 21, n. 2, abr.-jun., 2011 
Padrões para germinação, pureza, umidade e peso de mil sementes em análises de sementes ...

TABELA 7: Limites Baixo (B), Médio (M), Alto (A) e Muito Alto (MA) para a germinação (\%) de sementes de espécies florestais nativas. Santa Maria, RS. 2009.

TABLE 7: Low (B), Medium (M), High (A) and Very High (MA) Limits for germination (\%) in seeds of native tree species. Santa Maria - RS, 2009.

\begin{tabular}{cccccc}
\hline Limites & $\begin{array}{c}\text { Allophylus } \\
\text { edulis }\end{array}$ & $\begin{array}{c}\text { Apuleia } \\
\text { leiocarpa }\end{array}$ & $\begin{array}{c}\text { Cassia } \\
\text { leptophylla }\end{array}$ & $\begin{array}{c}\text { Cassia } \\
\text { multijuga }\end{array}$ & $\begin{array}{c}\text { Cedrela } \\
\text { fissilis }\end{array}$ \\
\hline $\mathrm{B}$ & $\mathrm{Y} \leq 47,56$ & $\mathrm{Y} \leq 59,70$ & $\mathrm{Y} \leq 42,05$ & $\mathrm{Y} \leq 45,59$ & $\mathrm{Y} \leq 75,41$ \\
$\mathrm{M}$ & $47,56<\mathrm{Y} \leq 96,44$ & $59,70<\mathrm{Y} \leq 100,00$ & $42,05<\mathrm{Y} \leq 79,45$ & $45,59<\mathrm{Y} \leq 100,00$ & $75,41<\mathrm{Y} \leq 100,00$ \\
$\mathrm{~A}$ & $96,44<\mathrm{Y} \leq 100,00$ & $100,00<\mathrm{Y} \leq 100,00$ & $79,45<\mathrm{Y} \leq 98,16$ & $100,00<\mathrm{Y} \leq 100,00$ & $100,00<\mathrm{Y} \leq 100,00$ \\
$\mathrm{MA}$ & $\mathrm{Y}>100,00$ & $\mathrm{Y}>100,00$ & $\mathrm{Y}>98,16$ & $\mathrm{Y}>100,00$ & $\mathrm{Y}>100,00$ \\
\hline \hline \multirow{2}{*}{ Limites } & Cupania & Enterolobium & Eugenia & Jacaranda \\
& vernalis & contortisiliquum & involucrata & micrantha \\
\hline $\mathrm{B}$ & $\mathrm{Y} \leq 8,00$ & $\mathrm{Y} \leq 83,37$ & $\mathrm{Y} \leq 68,78$ & $\mathrm{Y} \leq 81,15$ \\
$\mathrm{M}$ & $8,00<\mathrm{Y} \leq 58,00$ & $83,37<\mathrm{Y} \leq 100,00$ & $68,78<\mathrm{Y} \leq 93,22$ & $81,15<\mathrm{Y} \leq 94,85$ \\
$\mathrm{~A}$ & $58,00<\mathrm{Y} \leq 83,00$ & $100,00<\mathrm{Y} \leq 100,00$ & $93,22<\mathrm{Y} \leq 100,00$ & $94,85<\mathrm{Y} \leq 100,00$ \\
$\mathrm{MA}$ & $\mathrm{Y}>83,00$ & $\mathrm{Y}>100,00$ & $\mathrm{Y}>100,00$ & $\mathrm{Y}>100,00$ \\
\hline \hline \multirow{2}{*}{ Limites } & Lafoensia & Parapiptadenia & Peltophorum & Psidium \\
& pacari & rigida & dubium & cattleyanum \\
\hline $\mathrm{B}$ & $\mathrm{Y} \leq 66,67$ & $\mathrm{Y} \leq 89,98$ & $\mathrm{Y} \leq 74,41$ & $\mathrm{Y} \leq 67,29$ \\
$\mathrm{M}$ & $66,67<\mathrm{Y} \leq 93,33$ & $89,98<\mathrm{Y} \leq 100,00$ & $74,41<\mathrm{Y} \leq 99,59$ & $67,29<\mathrm{Y} \leq 85,71$ \\
$\mathrm{~A}$ & $93,33<\mathrm{Y} \leq 100,00$ & $100,00<\mathrm{Y} \leq 100,00$ & $99,59<\mathrm{Y} \leq 100,00$ & $85,71<\mathrm{Y} \leq 94,93$ \\
$\mathrm{MA}$ & $\mathrm{Y}>100,00$ & $\mathrm{Y}>100,00$ & $\mathrm{Y}>100,00$ & $\mathrm{Y}>94,93$ \\
\hline
\end{tabular}

Os padrões propostos para as variáveis pureza, peso de mil sementes e grau de umidade (Tabelas 4, 5 e 6) devem ser utilizados como parâmetros que auxiliam o entendimento dos valores propostos para os limites da germinação (Tabela 7). Essa variável possui maior importância dentro das análises de sementes e serve como valor base, que permite o controle de qualidade para avaliação dos lotes.

Neste estudo, merecem destaque as espécies Peltophorum dubium, pelo seu potencial na arborização urbana, na indústria moveleira e na construção civil em que é muito utilizada para confecção de pisos e parquets, e Psidium cattleyanum, bastante utilizada em reflorestamentos de áreas degradadas em função da rápida dispersão, realizada pela avifauna local, apreciadora de seus frutos. Para a primeira, o limite descrito como médio (M), na variável germinação, estão entre valores de 74,41 e 99,59\% (Tabela 7). Estudo realizado por Wielewicki et al. (2006), propondo padrões de germinação para espécies florestais nativas do Rio Grande do Sul, estabelece o valor de $79 \%$ como padrão mínimo de germinação para a mesma espécie. Assim, observa-se que o padrão de germinação proposto por Wielewicki et al. (2006) é mais rigoroso que o proposto no presente trabalho. Já para Psidium cattleyanum, o limite médio (M) apresentou valores entre 67,29 e 85,71\% (Tabela 7). Os resultados da germinação de Psidium cattleyanum apresentaram aderência à distribuição normal e, segundo Costa et al. (2002), quando há normalidade a utilização da mediana e do pseudossigma é equivalente à utilização da média e do desviopadrão. Por isso, os limites propostos Wielewicki et al. (2006) para essa espécie concordam com o obtido com o critério do Pseudossigma (COSTA et al., 2002).

Destaca-se que, para uma avaliação mais criteriosa e precisa, a tabela de classificação a ser utilizada deverá seguir o resultado obtido no teste de aderência à distribuição normal. Essa classificação deverá ser mais flexível, pois em se tratando de espécies florestais, a variável germinação normalmente é baixa, e dessa forma, uma classificação muito rigorosa poderá impossibilitar o estabelecimento de limites de confiabilidade, pois, possivelmente, não haverá índices germinativos reais que satisfaçam aos índices construídos com um critério muito rigoroso. 


\section{CONCLUSÕES}

Para uma classificação mais criteriosa e precisa, a tabela de classificação a ser utilizada deverá seguir o resultado obtido no teste de aderência à distribuição normal.

Lotes de sementes com valores de germinação (\%) dentro dos padrões classificados como baixos (B) devem ser descartados, pois não apresentam índices germinativos aceitáveis. Já os lotes de sementes, com porcentagem de germinação dentro dos limites de confiabilidade médio (M) ou alto (A), devem ser mantidos em armazenamento e análise.

As variáveis pureza, peso de mil sementes e teor de umidade devem ser utilizadas como auxiliares no entendimento da porcentagem de germinação.

\section{AGRADECIMENTO}

Ao $\mathrm{CNPq}$, pela concessão de bolsa de produtividade em pesquisa.

\section{REFERÊNCIAS BIBLIOGRÁFICAS}

BARBEDO, C. J. etal.Germinaçãoe armazenamento de diásporos de cerejeira (Eugenia involucrata DC. - Myrtaceae) em função do teor de água. Revista Brasileira de Sementes, Brasília, v. 20, n. 1, p. 184-188, 1998.

BRASIL. Ministério da Agricultura e Reforma Agrária. Secretaria Nacional de Defesa Agropecuária. Departamento Nacional de Defesa Vegetal. Regras para análise de sementes. Brasília, 1992. 365p.

CAMPOS, H. Estatística experimental nãoparamétrica. 4. ed. Piracicaba: Departamento de Matemática e Estatística/ESALQ, 1983. 349 p.

CARGNELUTTI FILHO, A. et al. Ajustes de funções de distribuição de probabilidade à radiação solar global no Estado do Rio Grande do Sul. Pesquisa Agropecuária Brasileira, Brasília,v. 39, n. 12, p. 1157-1166, dez. 2004.

CATALUNHA, M. J. et al. Aplicação de cinco funções densidade de probabilidade a séries de precipitação pluvial no estado de Minas Gerais.
Revista Brasileira de Agrometeorologia, Santa Maria, v. 10, n. 1, p. 153-162, 2002.

COSTA, N. H. et al. Novo método de classificação de coeficientes de variação para a cultura do arroz de terras altas. Pesquisa Agropecuária Brasileira, Brasília, v. 37, n. 3, p. 243-249, mar. 2002.

DURIGAN, G. et al. Sementes e mudas de árvores tropicais. 2. ed. São Paulo: Páginas \& Letras, 2002. $67 \mathrm{p}$.

FIGLIOLIA, M. B.; PIÑA-RODRIGUES, F. C. M. Considerações práticas sobre o teste de germinação. IF Série Registros, São Paulo, v. 14, p. 45-59, 1995. FORTES, F. O. et al. Agrupamento em amostras de sementes de espécies florestais nativas do Estado do Rio Grande do Sul - Brasil. Ciência Rural, Santa Maria, v. 38, n. 6, p. 1615-1623, 2008.

GARCIA, C. H. Tabelas para classificação do coeficiente de variação. Piracicaba: Instituto de pesquisas Florestais, 1989. 12 p. (Circular Técnica, 171).

GOULART, D. et al. Avanços na Análise de Sementes. Seed News, Pelotas, v. 12, n. 1, p. 12, 2008.

LÚCIO, A. D. et al. Classificação dos experimentos de competição de cultivares quanto a sua precisão. Pesquisa Agropecuária Gaúcha, Porto Alegre, v. 5, n. 1, p. 99-103, 1999.

LÚCIO, A. D. et al. Distribuição de probabilidade em análises nutricionais de espécies florestais. Revista Ceres, Viçosa, v. 54, n. 313, p. 214-224, 2007.

OLIVEIRA,E.C. etal.Propostas para a padronização de metodologias em análise de sementes florestais. Revista Brasileira de Sementes, Brasília, v. 11, n. 1/2/3, p. 1-42, 1989.

PINÃ-RODRIGUES, F. C. M.; VIEIRA, J. D. Teste de germinação. In: PINÃ-RODRIGUES, F. C. M. (Ed.). Manual de análise de sementes florestais. Campinas: Fundação Cargill, 1988. p.70-90.

VIEIRA, A. H. et al. Técnicas de produção de sementes florestais. Boa Vista: EMBRAPA-CPAF, Rondônia, 2001, p.1-4. (Documentos; 205).

WIELEWICKI, A. P. et al. Proposta de padrões de germinação e teor de água para sementes de algumas espécies florestais presentes na região sul do Brasil. Revista Brasileira de Sementes, Pelotas, v. 28, n. 3, p. 191-197, 2006. 